\title{
New technology in echocardiography I: Doppler techniques
}

\author{
W Norman McDicken, Peter R Hoskins, Carmel M Moran, George R Sutherland
}

Ultrasonic technology is in several ways ideally suited to cardiac research and to routine clinical examinations. It provides truly real time images and detailed information on moving anatomical structures and blood. The portable nature and relatively low cost of the equipment makes it well suited to use in hospitals. Ultrasonic technology for cardiology is still developing and in recent years this is occurring on diverse fronts such as transoesophageal transducers, catheter transducers, contrast agents, and Doppler imaging. The aim of this review is to assist the clinical user in keeping abreast of the technological advances.

\section{Transducers}

The variety in transducer design gives great flexibility to clinical applications of ultrasound. Below an ultrasonic frequency of $10 \mathrm{MHz}$, transducers normally have ceramic piezoelectric elements (crystals), above $10 \mathrm{MHz}$ the required thinness of such elements makes them brittle. However, some high frequency ceramic devices are found but in addition piezoelectric plastic elements are available. At present $30 \mathrm{MHz}$ is commonly used in small ultrasonic imaging catheters. Recently transducers having elements made from a composite of ceramic and plastic have been made which operate over a wider range of frequencies than normal for diagnostic devices. ${ }^{1}$ These wideband transducers can generate shorter pulses and also transmit at one frequency range, say 2 to $4 \mathrm{MHz}$, and receive on another, say 4 to $8 \mathrm{MHz}$. It will be seen later that this feature is employed in new harmonic Doppler techniques being used to detect contrast agents.

Real time pulse-echo transducers sweep the beam in a plane to produce a two dimensional scan. There is considerable interest in transducers which can alter the plane-of-scan without moving the whole transducer assembly, particularly for transoesophageal imaging and also for three dimensional imaging both inside and outside the body. In commercially available omniplane scanners this is done by physically moving the crystal elements. ${ }^{2}$ However, just as a one dimensional array of elements in a conventional phased array can produce a two dimensional scan, a two dimensional array of elements can perform a three dimensional scan (fig 1). Such devices are still in the prototype stage of development. ${ }^{3}$

Doppler blood flow techniques

Doppler techniques provide us with more detailed information on blood flow within the body than any other technique. Detailed information in real time on the velocities of moving blood cells at selected sites is presented on sonograms ${ }^{4}$ while regions of blood motion in relation to the surrounding anatomy are provided by colour flow imaging. Both of these techniques are still developing.

\section{THE SONOGRAM (SPECTOGRAM, SPECTRAL} DOPPLER)

In a sonogram at each instant Doppler velocities (shift frequencies) related to the selected site are displayed in real time and represent a picture of all the Doppler information present in the reflected ultrasound (fig 2A). The blackness of the trace at any point in this display is related to the amount of blood flowing at the corresponding velocity. For example sonograms of blood passing through the mitral valve will demonstrate a strong band around the top of the flow pattern and this is associated with the presence of uniformly high velocities of blood within the Doppler sample volume. Where there is a range of blood velocities within the sample volume the sonogram will show a broader range of Doppler shifts. Inspection of a vertical single spectral line from a sonogram shows that in fact there is considerable variation of the magnitude of the Doppler trace (fig 2B). This variation is called "Doppler speckle" and arises as a direct result of the technique of fast Fourier transform (FFT) analysis used for estimation of the Doppler frequencies. Techniques have been described for reducing the degree of Doppler speckle-for example, averaging of several Doppler waveforms (fig 2C and D) - and the use of alternative procedures such as autoregression analysis to estimate the

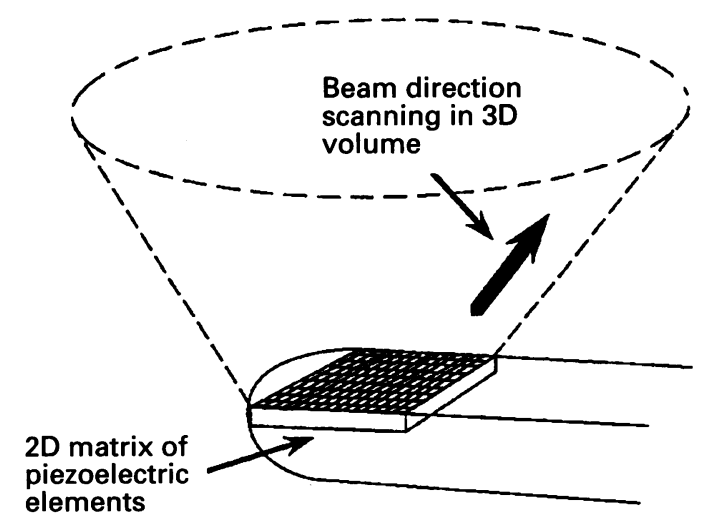
elements

Figure 1 A two dimensional electronic array capable of scanning the ultrasonic beam in three dimensional space without movement of the transducer elements. 
Figure 2 Frequency spectra. (A) Plot of frequency components $(F)$ versus time $(T)$ producing a sonogram. (B) Plot of power $(P)$ of each frequency component versus frequency $(F)$ producing an

instantaneous spectrum. Spectra presented as adjacent vertical grey shade lines produce a sonogram. (C) Smoothed sonogram by averaging over heart cycles. (D) Smoothed spectrum by averaging over heart cycles.

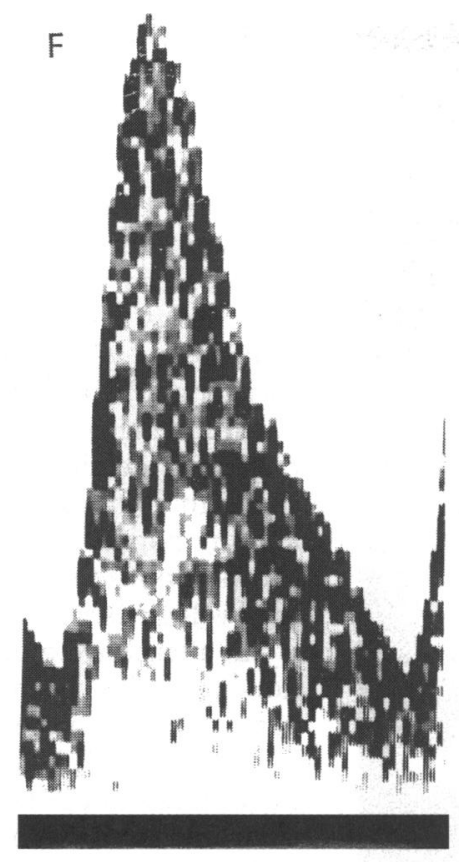

A

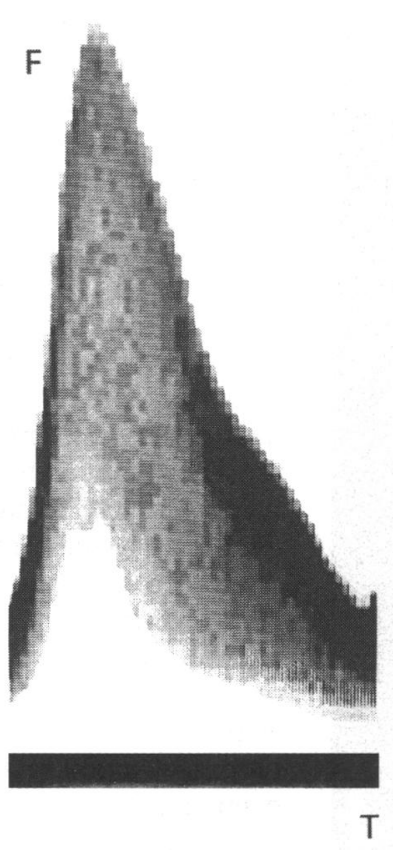

C

Doppler spectrum. ${ }^{5}$ The latter can also improve the temporal resolution of the sonogram for example, $1 \mathrm{~ms}$ compared with $10 \mathrm{~ms}$ for the FFT method. However, the FFT based sonogram has proven satisfactory for many purposes and is still the commonest type.

Recently zero-crossing detectors for the estimation of mean frequency have shown a reemergence in the field of intravascular ultrasound. This is basically obsolete technology which suffers from well documented problems. ${ }^{6-8}$ These include:

- The estimated mean frequency is strongly dependent on the velocity profile

- Electronic noise and wall thump cause significant errors in mean frequency estimation

- The output must be smoothed, which means that rapidly changing velocities are not recorded accurately

- For blood flowing simultaneously in both the forward and reverse direction the zerocrossing detector output has no meaning. The same conclusions are being drawn by more recent studies. ${ }^{9}$

\section{COLOUR FLOW IMAGING}

The development of Doppler methods for cardiology saw the progression from stand-alone pulsed wave and continuous wave devices through duplex B mode plus Doppler to colour flow imaging systems. Therefore although duplex units have been of value they were essentially stepping stones to colour systems, which are much better suited to routine use. Virtually all colour flow scanners use a process called autocorrelation detection to estimate the 


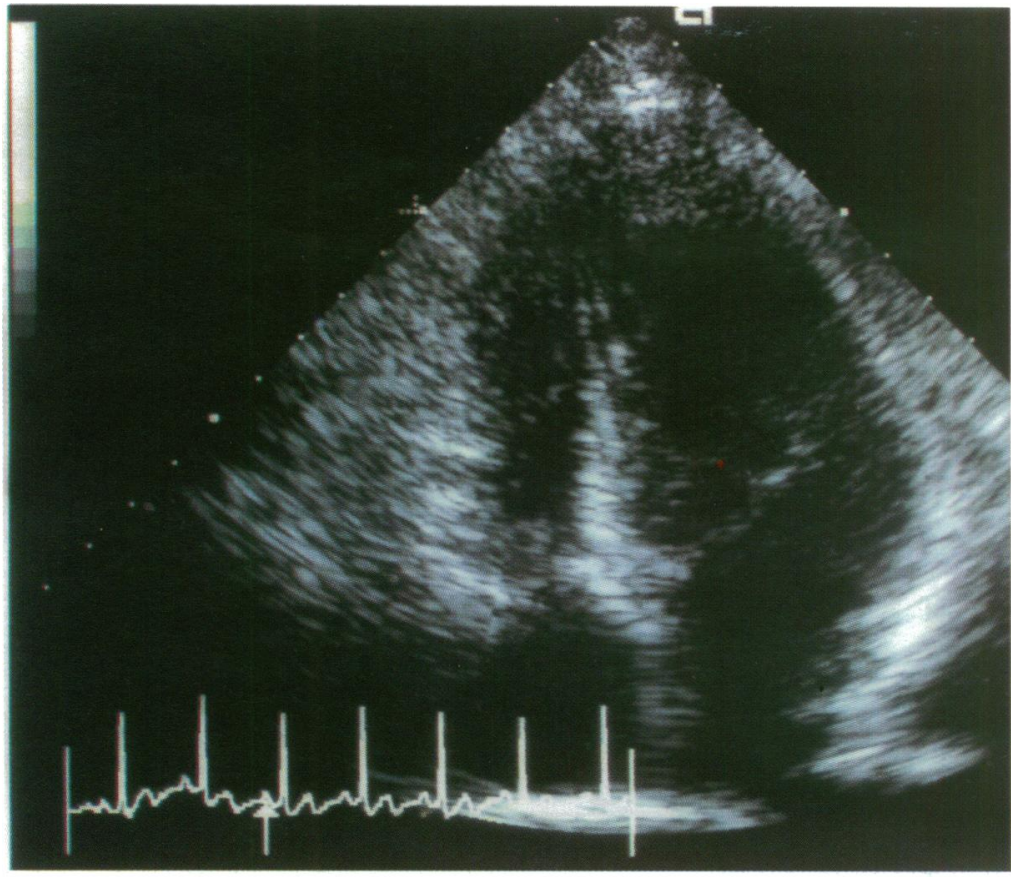

$A$

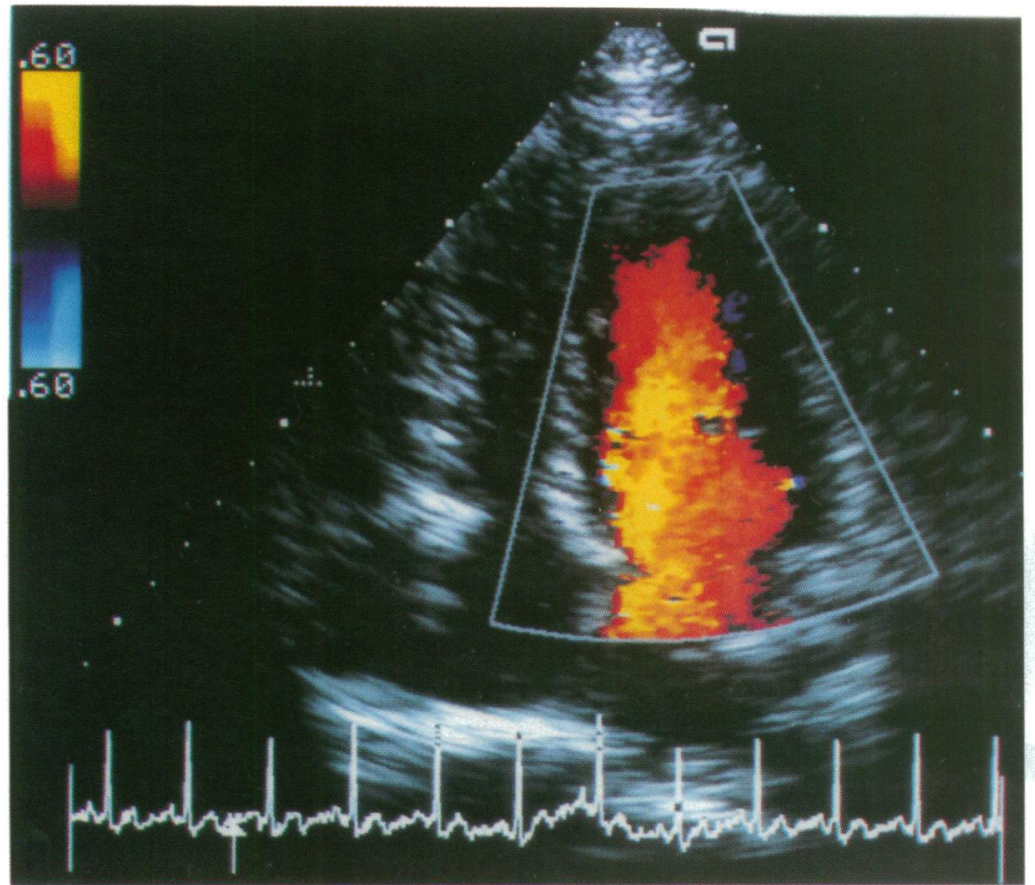

$B$

Figure 3 (A) Conventional B mode echo image. (B) Colour flow Doppler image of velocity components in the cardiac chamber.

mean Doppler frequency shift for each image pixel. The detailed signal processing aspects of this method are described in detail elsewhere. ${ }^{10}$ Essentially this method compares the relative phases of several consecutive echoes from a moving target. Moving targets such as blood are associated with a change in the phase of consecutive echoes whereas stationary targets have no phase change. To estimate mean Doppler shift this process requires just a few ultrasound pulses per scan line, typically 3-10 (compared with 50-100 for the production of a single spectral line of a sonogram), and this makes it possible to move the ultrasound beam reasonably quickly to produce a real time image.

A colour flow image has the same constraints as a sonogram; the images are apt to aliase and are angle dependent-that is, the displayed colour will depend on the angle between the ultrasound beam and the direction of the blood flow. One commercial company has developed a colour flow system which attempts to raise the velocity at which aliasing occurs (Kontron, Switzerland). In this system two distinct transmit frequencies are used. Though there are no published details on the technology, comparison with continuous wave Doppler in flow rigs and in patients suggests that this technique shows promise for the unambiguous display of high velocities. ${ }^{11}$

An alternative method of producing a colour flow image has been described ${ }^{12}$ and forms the basis of one commercial scanner (Philips, The Netherlands). It relies on tracking in real time the movement of the pattern of low level echoes from one dimensional A-scan images of blood within vessels or cardiac chambers. This is commonly referred to as "speckle tracking" or "time domain processing". To truly track the echo pattern of blood would require a two dimensional approach, and this also has been described, ${ }^{13}$ whereas the commercial system (Philips) tracks the echo pattern along the beam axis only. Such a one dimensional system has a higher colour scan line density than is possible with conventional autocorrelator based colour Doppler; however. it also suffers from beam-velocity angle dependence, which a two dimensional tracking system theoretically does not. The two dimensional tracking system has been used to image flow in arteries: its use in the heart where there are high velocities and turbulent flow remains the subject of research. Tracking systems may also be of value in the study of tissue velocities within the myocardium.

The two colour flow methods above both provide images in which an estimate of velocity is made. In addition the power or energy of the Doppler signal may be displayed. This has been available on some ultrasound scanners for several years and has been used in research studies which have attempted to improve the assessment of high velocity jets associated with valve stenosis ${ }^{14}{ }^{15}$; it did not, however, show great advantage over the standard mean velocity colour image in the depiction of flow. Recently the technique has been modified and currently there is great interest because it seems that low velocity flow and flow in small vessels can be depicted with considerably improved sensitivity. ${ }^{16}$ Power Doppler by its very nature provides no information concerning the speed or direction of the flow, and this in fact may be advantageous in applications in which information on the presence only of flow is needed. The displayed power Doppler image produced by the more recent systems uses averaging over several scans. This helps reduce the image noise and enhance the visualisation of small vessels. None the less, it is likely that this procedure is inappropriate in cardiology where there is considerable interest 


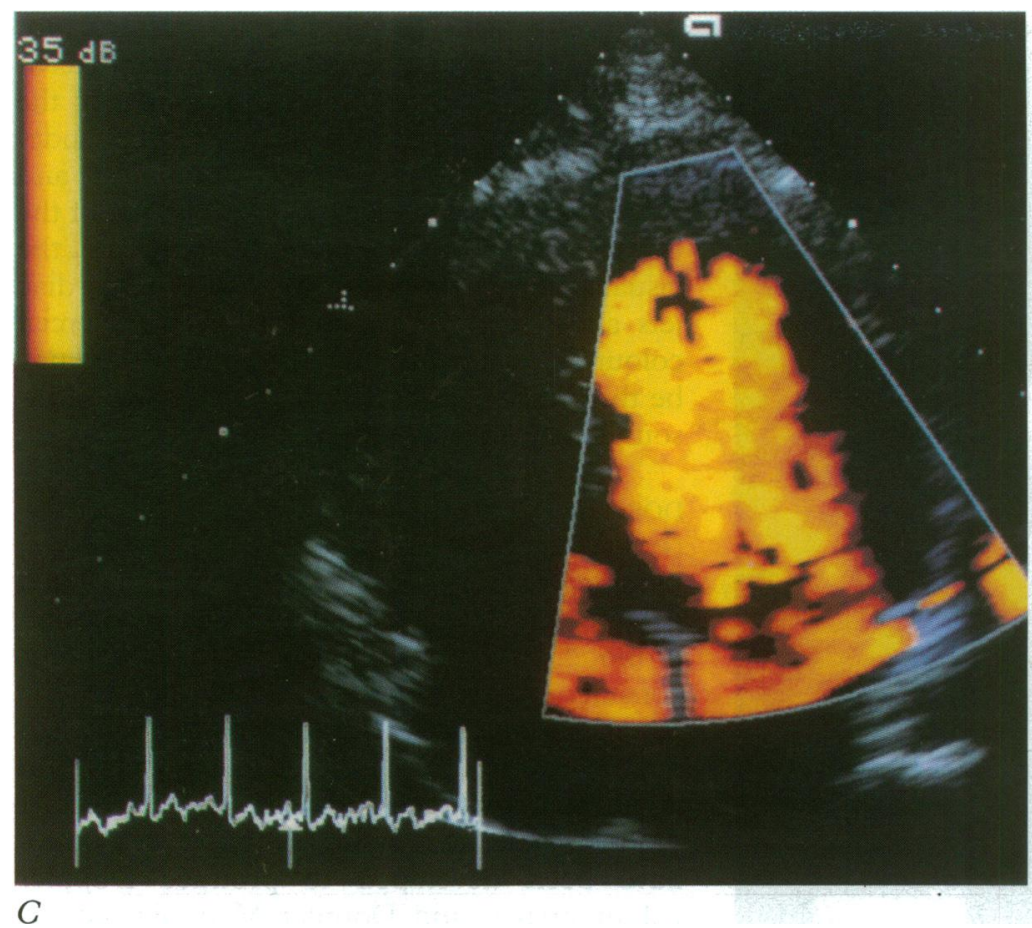

Figure 3 (C) Colour image of the power of the Doppler signal from regions of the cardiac chamber.

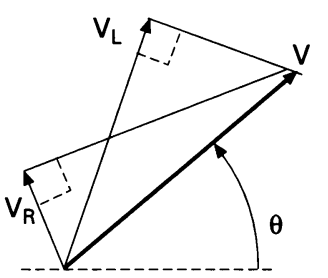

Figure 4 Velocity components $V_{L}$ and $V_{R}$ of the true velocity $V$ as measured from the $L$ and $R$ beam directions. cycle. The use of power Doppler in cardiology has yet to be critically evaluated (fig 3 ).

VECTOR DOPPLER TECHNIQUES

For the single beam Doppler systems described above the velocity information is related only to the component of velocity which is along the direction of the ultrasound beam. This leads to the dependence of the spectral Doppler velocity and of the colour flow mean velocity image on in the details of flow throughout the cardiac

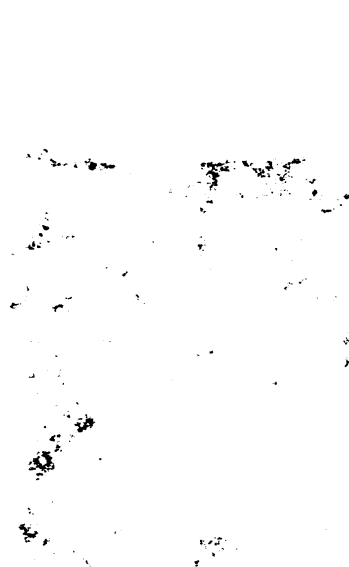

A
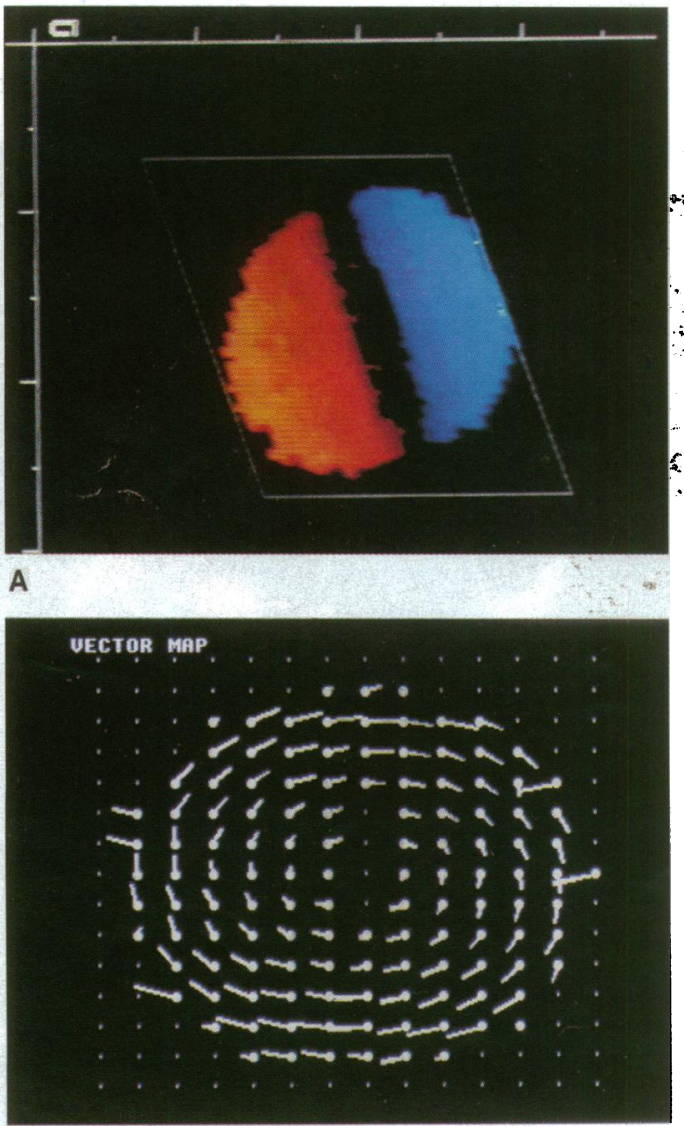

C the angle between the beam and the flow direction. Errors can arise in the estimation of the maximum velocity of an intracardiac jet from a sonogram when the angle correction cannot be made. Visualisation of a jet is also difficult in a colour flow image when the jet flows at right angles to the beam.

To fully describe a velocity in magnitude and direction, a velocity vector is required. This vector consists of three components of velocity at right angles to each other. If the flow is confined to a single plane, however, two components are sufficient. There are systems for in-plane flow which attempt to produce angleindependent velocity measurements and colour images by measuring the components of the velocity vector. ${ }^{17-21}$ The colour image technique needs estimates of the components of velocity $V_{L}$ and $V_{R}$ along two separate directions (fig 4). These are added to produce an estimate of the velocity magnitude and direction in the scan plane. Figure 5 shows images taken using a rotating phantom. This consists of a disc of foam which is driven in a circular fashion at constant speed. The colour flow images show equal halves of blue and red which is consistent with motion away from the transducer for one half of the phantom and motion towards the transducer for the other half of the phantom. Corresponding points in each image form the estimates of velocity components $V_{L}$ and $V_{R}$. These are compounded to give the magnitude and direction of the velocity at each point in the scan plane. A vector map may be produced in which the directions of motion are described by the directions of arrows, and the length of each arrow is proportional to the magnitude $\mathrm{V}$ of the velocity. Techniques which attempt to estimate all three components of the velocity vector are

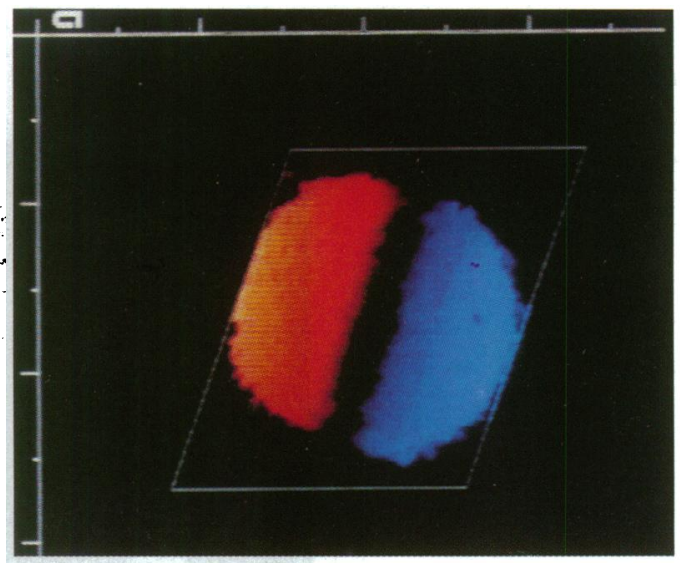

B
Figure 5 ( $A$ ) and $(B)$ two colour component images obtained from two different beam directions. (C) Resultant velocity vector map constructed from the velocity component image data. 


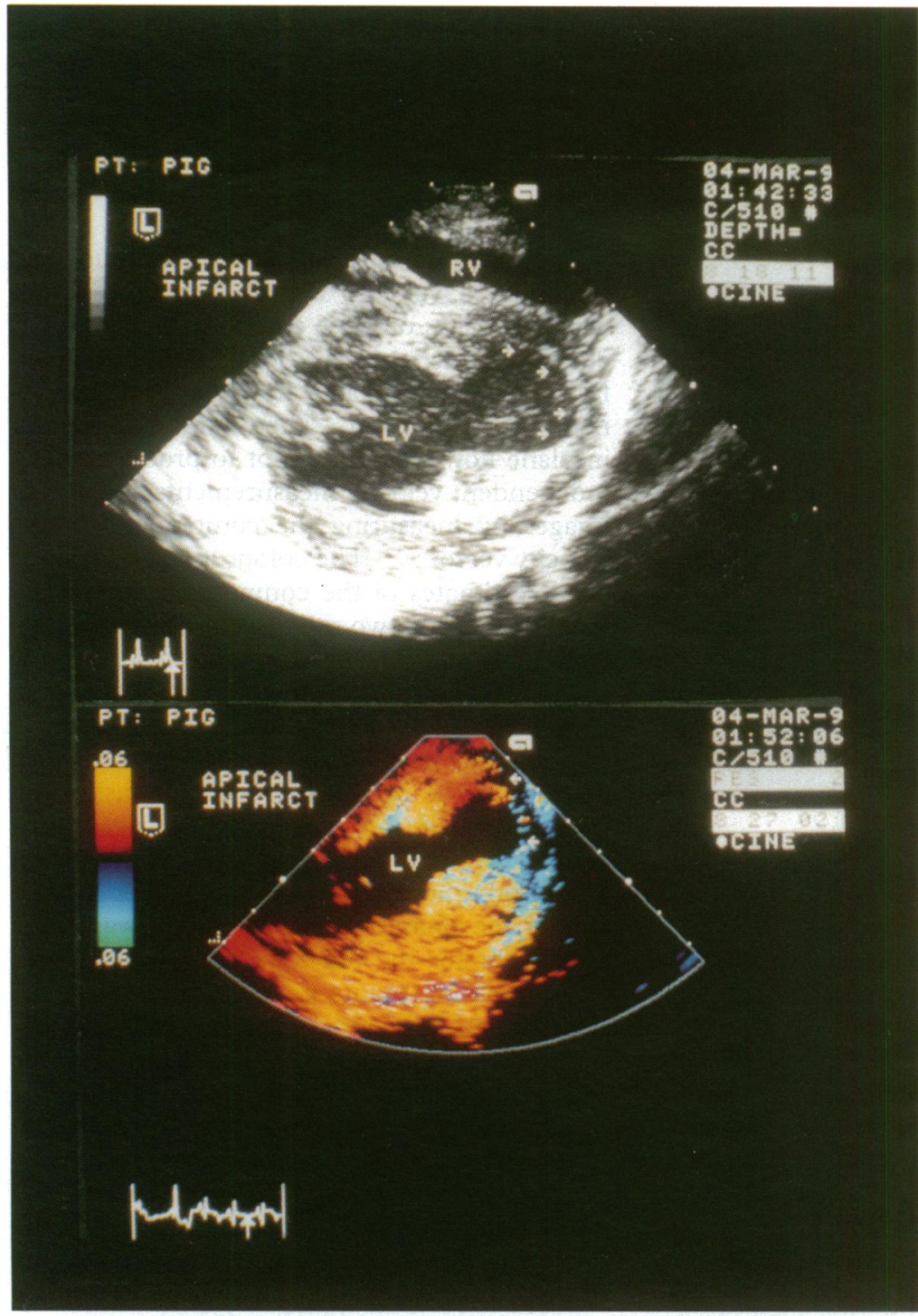

Figure 6 Grey shade pulse-echo image and corresponding Doppler tissue image (DTI). The DTI depicts the Doppler velocity components and their direction relative to the transducer. likely to remain the domain of research for some time to come. On the other hand techniques which estimate two components of the velocity vector, in order to produce angle-independent maximum velocity estimation and colour flow images within the scan plane of the transducer, have potential in relation to the visualisation and velocity estimation of cardiac jets and may find their way into commercial colour flow systems. One limiting factor may be the difficulty in finding access for two ultrasound beams into the heart.

DOPPLER IMAGING OF MYOCARDIAL VELOCITIES (DOPPLER TISSUE IMAGING)

Doppler techniques have until recently been used to detect, image, and quantify the motion of blood. In cardiac disease, however, there is considerable interest in the motion of the myocardium, both the overall motion and activity within the muscle. The overall motion is normally studied using $B$ mode and $M$ mode pulse-echo imaging. Doppler techniques have been developed to produce Doppler colour images and Doppler $M$ mode recordings of tissue motion. This was initially done by manipulating the control settings of a standard colour Doppler blood velocity imager. ${ }^{22}$ In particular, a small velocity range was selected $( \pm 6 \mathrm{~cm} / \mathrm{s})$, the high-pass wall thump filter was set low to allow slow tissue motions to be detected $(92 \mathrm{~Hz})$ and the Doppler gain was reduced such that tissue signals had a magnitude similar to that normally encountered from blood.

A series of assessments with moving tissue test-objects showed that motion of tissue type materials could be reliably imaged with these machine settings. Later proprietary software was obtained which enabled the Doppler tissue imaging mode to be entered directly (Acuson, USA). Examples of Doppler tissue
Figure 7 Transverse Doppler tissue image of left ventricle.

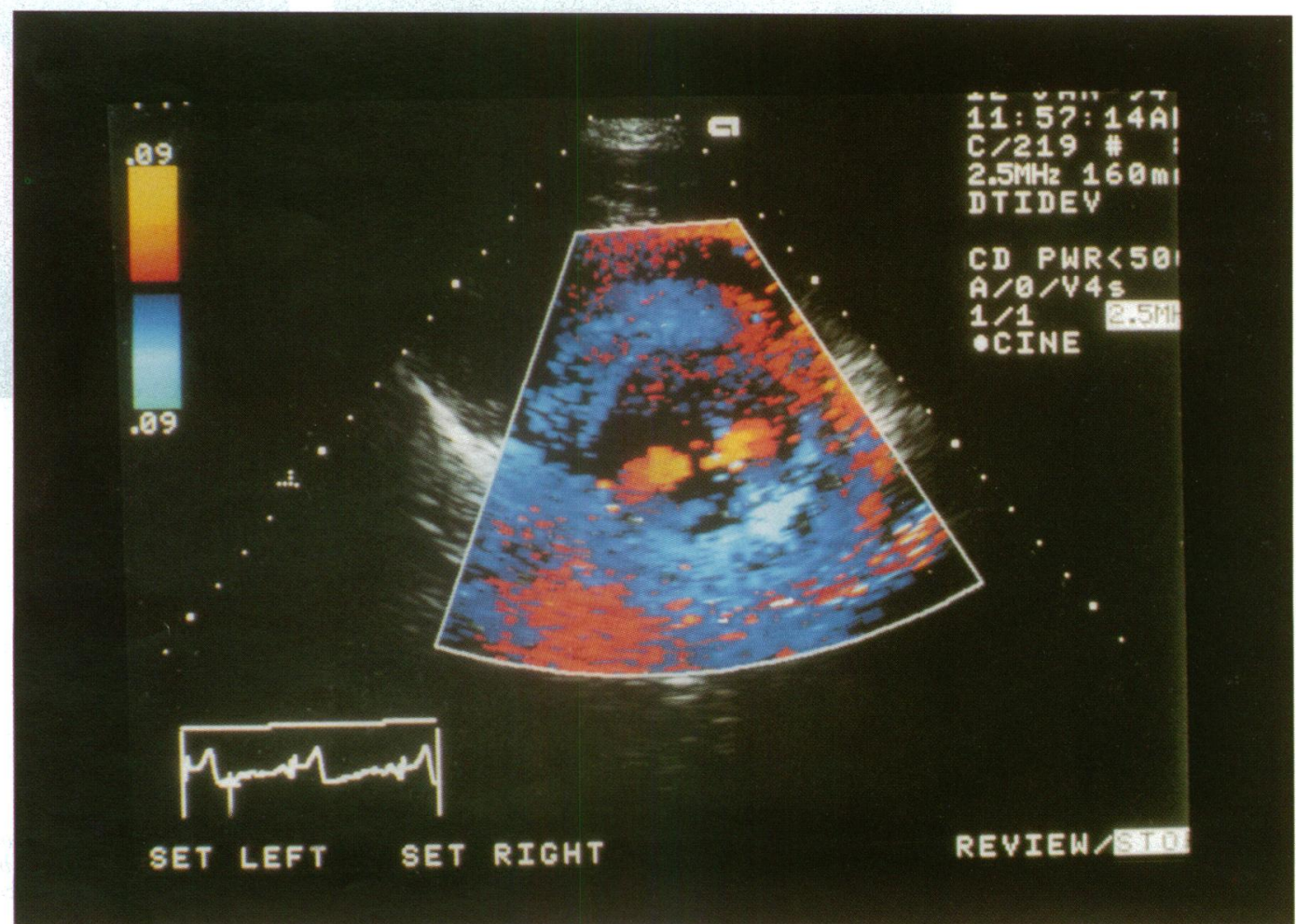




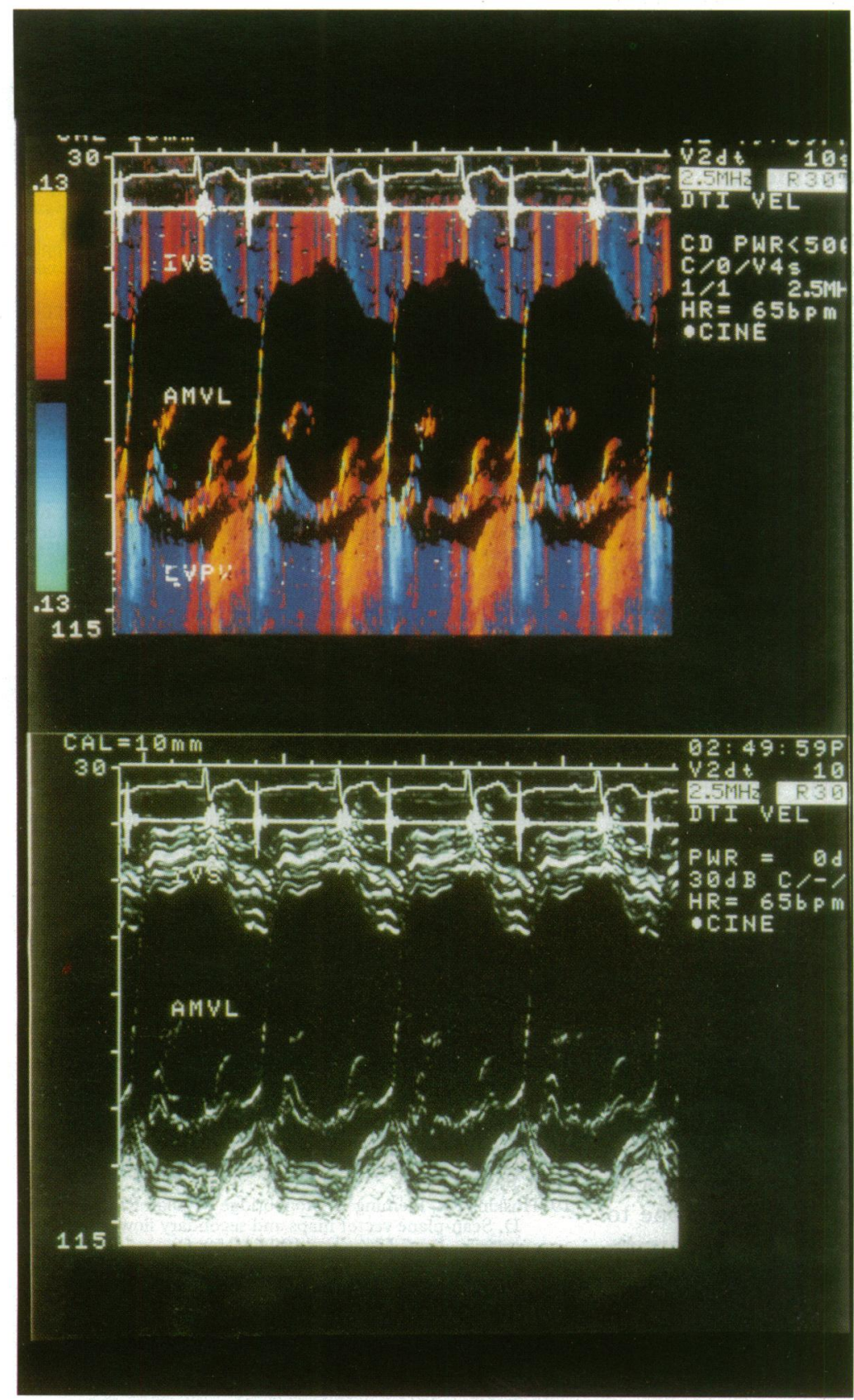

Figure 8 Grey shade pulse-echo $M$ mode trace and corresponding Doppler $M$ mode trace.

images are presented in figures 6 and 7, like blood flow images, red colour-coding represents motion toward the transducer whereas blue denotes motion away: The Doppler $M$ mode provides detailed temporal information on variations in velocity across the myocardium (fig 8). A number of investigations are now underway on animals, normal volunteers, and patients to establish the value of this new technology. ${ }^{23-26}$ Several features of the method point to it becoming a useful tool:

- Velocities and velocity gradients within the myocardium can be measured rather than the overall motion normally studied with the established $B$ and $M$ mode techniques.

- Information on tissue characterisation relating to the myocardium is contained in the ultrasonic frequency rather than the amplitude of the echo and is therefore much less prone than pulse-echo methods to distortion by tissue layers between the transducer and the heart muscle.
- Inert material which is not stretching or shrinking, as might be expected for infarcted tissue, has the same velocity component along each scan line. That is to say, in this situation each scan line has a particular colour depending on the velocity component for that line. Infarcted tissue therefore usually appears as a uniformly coloured region of low velocity. Material which is actively changing its shape shows a range of colours along each scan line that is, velocity gradients are detected.

- Tissue boundaries need not be depicted sharply for velocity measurements to be made, as is the case when rate of change of wall thickness is being measured from an $M$ mode scan.

A number of laboratory studies have been carried out to assess the performance of the present system and to ensure that it could handle tissue type signals rather than those from blood. Blood produces a smaller range of echo amplitudes - that is, a smaller dynamic rangethan those from tissues. Tissue equivalent materials in the form of a rotating $10 \mathrm{~cm}$ diameter disc and oscillating slabs containing grooves of different size were used in these tests. It was shown that velocities could be measured reliably, as for blood flow, provided that the signal was not allowed to saturate by having the system sensitivity too high. The aliasing artifact was encountered at the expected velocities. The spatial resolution, determined by the minimum size of square groove which could be identified, was around $2.5 \mathrm{~mm}$. This is poorer than desired but sufficient to allow clinical studies to proceed. A further check on performance was the comparison of velocity gradients in the myocardium measured by Doppler $M$ mode and rate of change of wall thickness by pulse-echo $M$ mode. Although these techniques do not measure the same quantity, there was good temporal agreement in the peaks of their values. ${ }^{25}$

The present research is essentially being undertaken with machines which are a modification of those produced for blood flow imaging and are therefore first generation Doppler tissue imaging devices. In the future, improved spatial resolution and more complete imaging of the heart chamber walls, particularly the lateral walls, should be possible. The dependence of the velocity components depicted in an image on the angle between the ultrasound beam and the direction of tissue motion may be overcome, for example, by using the two dimensional tracking methods mentioned above. Doppler tissue images are improved by the use of microbubble contrast agents injected into the coronary arteries. The mechanism of the enhancement is not yet clearly understood, it may be due to the agent moving through arterioles in the muscle or to agent attached to the muscle. Both mechanisms would enable perfusion to be studied, the second would also help with the measurement of velocities in the myocardium. Developments in contrast agents are progressing rapidly and could have significant impact on Doppler tissue motion techniques. The portability of the 
equipment will be improved to enable it to be used for serial assessment of patients at the bedside.

\section{Intravascular Doppler techniques}

Though intravascular Doppler is much less commonly used than intravascular B scan imaging, it is commercially available. Typically a $12 \mathrm{MHz}$ Doppler transducer is mounted on the end of the guidewire, enabling the Doppler beam to point forward in the direction of the wire axis. In practice the guidewire partially blocks the lumen and there is a region of turbulent flow beyond the end of the wire. It is desirable to acquire Doppler waveforms from blood flowing some distance downstream from the region of turbulence, and for this reason pulsed wave ultrasound is used with the sample volume set about $5 \mathrm{~mm}$ from the crystal surface. This is adequate in normal vessels of $3-4 \mathrm{~mm}$ diameter where the Doppler wire represents a $1-2 \%$ narrowing of the cross sectional area; in a tight $1 \mathrm{~mm}$ diameter stenosis, however, the Doppler wire represents a $25 \%$ reduction in area, and it is likely that the Doppler waveforms will be influenced by the partial blocking of the vessel lumen. Intravascular Doppler techniques have largely been used in research studies, for example in an attempt to estimate myocardial perfusion reserve by recording Doppler waveforms before and after infusion of an active vasodilator such as papaverine. ${ }^{27} 28$

Intravascular colour flow imaging is possible in theory, but it is technically difficult to make miniature scanners for which the plane-of-scan looks forward down the vessel. Since the scanning beam would make small angles to the direction of flow, the high values of velocity components may make such imagers prone to aliasing. Scanners in which the beam is perpendicular to the direction of flow, as used to examine artery walls, produce poor Doppler signals.

\section{Three dimensional Doppler imaging}

$3 \mathrm{D}$ reconstruction of colour flow images is feasible if the scanning beam is made to sweep through the volume of interest and velocity information is gathered from a large number of beam directions. This requires collection of a series of colour flow images which have all been acquired at the same point in the cardiac cycle, so that cardiac gating of the colour flow images is required. At present the technique is the subject of research, and to date reports have concentrated on 3D reconstruction of arterial colour flow images; for example, the existence of helical flow patterns in the internal carotid artery has been demonstrated..$^{29}$

1 Gururaja TR, Schulze WA, Cross LE, Newham RE. Piezoelectric composite materials for ultrasonic transducer Piezoelectric composite materials for ultrasonic transducer applications. Part 11: Evaluation of ultrasonic medical applications.

2 Souquet J, Hanrath P, Zitelli L, Kremer R, Langenstein RA,
Schulter M. Transesophageal phased array for imaging the heart. IEEE Trans Biomed Eng 1982;29:707-12.

3 von Ramm OT, Smith SW, Pavy HG. High-speed ultrasound volumetric imaging system. Part 1 Transduce design and beam steering. IEEE Trans Ultrasons Ferroelect Freq Contr 1991;38:100-8.

4 McDicken WN. Diagnostic ultrasonics: principles and use of instruments. Edinburgh: Churchill Livingstone, 1991.

5 Schlindwein FS, Evans DH. A real time autoregressive spectrum analyser for Doppler ultrasound signals. Ultrasound Med Biol 1989;15:263-72.

6 Flax SW, Webster JG, Updike SJ. Pitfalls using Doppler ultrasound to transduce blood flow. IEEE Trans Biomed Eng 1973;20:306-9.

7 Lunt MJ. Accuracy and limitations of the ultrasonic Doppler blood velocimeter and zero crossing detector. Utrasound Med Biol 1975;2:1-10.

8 Johnstone KW, Maruzzo BC, Cobbold RSC. Errors and artifacts of Doppler flowmeters and their solution. Arch Surg 1977;112:1335-42.

9 Sudhir K, Hargrave VK, Johnson EL, Aldea G, Mori H, Ports TA, Yock PG. Measurement of volumetric coronary flow with a catheter: validation in an animal model. $\mathrm{Am}$ Heart $₹$ 1992;124:870-5.

10 Kasai C, Namekawa K, Koyano A, Onoto R. Real time two dimensional blood flow using an autocorrelation technique. IEEE Trans Sonics Ultrasonics 1985;SU-32.
nimention 458-64.

11 Fan P, Nanda NC, Cooper JW, Cape E, Yogananthan A. Color Doppler assessment of high flow velocities using a new technology: in vitro and clinical studies. Echocardiography 1990;7:763-9.

12 Bonnefous O, Pesque P. Time domain formulation of pulse Doppler ultrasound and blood velocity estimation by cross correlation. Ultrason Imag 1986;8:75-85.

13 Bohs LN, Friemel BH, McDermott BA;; Trahey GE. A real time system for quantifying and displaying two-dimensional velocities using ultrasound. Ultrasound Med Biol 1993;19:751-61.

14 Sahn DJ. Instrumentation and physical factors related to visualisation of stenotic regurgitant jets by Doppler color flow mapping. $\mathcal{F} \mathrm{Am}$ Coll Cardiol 1988;12:1354-65.

15 Simpson IA, Valdes-Cruz LM, Sonn DJ, Murillo A, Tamora $T$, Chung K. Doppler color flow mapping of simulated in vitro regurgitant jets: evaluation of the effects of orifice size and hemodynamic variables. $7 \mathrm{Am}$ Coll Cardiol 1989 13:1195-207.

16 Rubin JM, Bude RO, Carson PL, Bree RL, Adler RS. Power Doppler US: a potentially useful alternative to mean frequency based color Doppler US. Radiology 1994;190: 853-6.

17 Fei DY, Fu CT, Brewer WH, Kraft KA. Angle independent Doppler color imaging: determination of accuracy and a method of display. Ultrasound Med Biol 1994;20:147-55.

18 Maniatis TA, Cobbold RSC, Johnstone KW. Two-dimensional velocity reconstruction strategies for colour flow Doppler ultrasound images. Ultrasound Med Biol 1994; 20:137-45.

19 Hoskins PR, Fleming A, Stonebridge P, Allan PL, Cameron $D$. Scan-plane vector maps and secondary flow motions in arteries. Eur f Ultrasound 1994;1:159-69.

20 Fox MD, Gardiner WM. Three dimensional Dopple velocimetry of flow jets. IEEE Trans Biomed Eng 1988; BME-35:834-41.

21 Overbeek JR, Beach KW, Strandness DE. Vector Doppler: accurate measurement of blood velocity in two dimenions. Ultrasound Med Biol 1992;18:19-31.

22 McDicken WN, Sutherland GR, Moran CM, Gordon LN. Colour Doppler imaging of the myocardium. Ultrasound Med Biol 1992;18:651-4.

23 Moran CM, McDicken WN, Groundstroem KWE Sutherland GR. Potential applications of color-Doppler imaging of the myocardium in assessing contractility and perfusion. In: Nanda NC, Schlief R, eds. Advances in echo imaging using contrast enhancement. Dortrecht, The Netherlands: Kluwer; 1993:359-74.

24 Groundstroem KWE, Sutherland GR, Moran CM McDicken WN. Myocardial imaging by color-Doppler coded velocity mapping - from regional contraction to tissue characterisation? In Nanda NC, Schlief R, eds. Advances in echo imaging using contrast enhancement. Dortrecht, The Netherlands:Kluwer; 1993:375-99.

25 Fleming AD, Xia X, McDicken WN, Sutherland GR, Fenn L. Myocardial velocity gradients detected by Dopple imaging. Br 7 Radiol 1994;67:679-88.

26 Sutherland GR, Stewart MJ, Groundstroem KWE, Moran CM, Fleming A, Guell-Peris FJ, Riemersma RA, Fenn LN, Fox KAA, McDicken WN Colour Doppler myocarLN, Fox KAA, McDicken WN. Colour Doppler myocarmyocardial function. $\mathrm{f} \mathrm{Am} \mathrm{Soc} \mathrm{Echo} \mathrm{1994;7:441-58.}$

27 Cole JS, Hartley CJ. The pulsed Doppler coronary catheter: preliminary report of a new technique for measuring rapid changes in coronary artery flow velocity in man. changes in coronary arter.

28 Hartley CJ. Review of intracoronary Doppler catheters. Int $\mathcal{F}$ Card Imag 1989;4:159-68.

29 Picot PA, Rickey DW, Mitchell R, Rankin RN, Fenster A. Three-dimensional colour Doppler imaging. Ultrasound Med Biol 1993;19:95-104. 